Please do not remove this page

RMIT

UNIVERSITY

\title{
A genetic algorithm with local search for solving single-source single-sink nonlinear non-convex minimum cost flow problems
}

Ghasemishabankareh, Behrooz; Ozlen, Melih; Li, Xiaodong; Deb, Kalyanmoy

https://researchrepository.rmit.edu.au/esploro/outputs/9921860851101341/filesAndLinks?institution=61RMIT_INST\&index=null

Ghasemishabankareh, B., Ozlen, M., Li, X., \& Deb, K. (2020). A genetic algorithm with local search for solving single-source single-sink nonlinear non-convex minimum cost flow problems. Soft Computing, 24, 1153-1169. https://doi.org/10.1007/s00500-019-03951-2

Document Version: Accepted Manuscript

Published Version: https://doi.org/10.1007/s00500-019-03951-2

Repository homepage: https://researchrepository.rmit.edu.au

(c) 2019, Springer-Verlag GmbH Germany, part of Springer Nature.

Downloaded On 2023/04/26 15:36:04 +1000 
Thank you for downloading this document from the RMIT Research Repository.

The RMIT Research Repository is an open access database showcasing the research outputs of RMIT University researchers.

RMIT Research Repository: http://researchbank.rmit.edu.au/

\section{Citation:}

Ghasemishabankareh, B, Ozlen, M, Li, X and Deb, K 2020, 'A genetic algorithm with local search for solving single-source single-sink nonlinear non-convex minimum cost flow problems', Soft Computing, vol. 24 , pp. 1153-1169.

See this record in the RMIT Research Repository at:

https://researchbank.rmit.edu.au/view/rmit:57315

Version: Accepted Manuscript

Copyright Statement:

(C) 2019, Springer-Verlag GmbH Germany, part of Springer Nature.

\section{Link to Published Version:}

http://dx.doi.org/10.1007/s00500-019-03951-2 


\title{
A Genetic Algorithm with Local Search for Solving Single-Source Single-Sink Nonlinear Non-Convex Minimum Cost Flow Problems
}

\author{
Behrooz Ghasemishabankareh · Melih \\ Ozlen · Xiaodong Li · Kalyanmoy Deb
}

Published online : 20 March 2019

\begin{abstract}
Network models are widely used for solving difficult real-world problems. The minimum cost flow problem (MCFP) is one of the fundamental network optimisation problems with many practical applications. The difficulty of MCFP depends heavily on the shape of its cost function. A common approach to tackle MCFPs is to relax the non-convex, mixed-integer, nonlinear program (MINLP) by introducing linearity or convexity to its cost function as an approximation to the original problem. However, this sort of simplification is often unable to sufficiently capture the characteristics of the original problem. How to handle MCFPs with non-convex and nonlinear cost functions is one of the most challenging issues. Considering that mathematical approaches (or solvers) are often sensitive to the shape of the cost function of non-convex MINLPs, this paper proposes a hybrid genetic algorithm (GA) with local search (namely GALS) for solving single-source single-sink nonlinear non-convex MCFPs. Our experimental results demonstrate that GALS offers highly competitive performances as compared to those of the mathematical solvers and a standard genetic algorithm.
\end{abstract}

Keywords Minimum cost flow problem · Non-convex cost function - Genetic algorithm · Local search

B. Ghasemishabankareh · M. Ozlen · X. Li

School of Science, RMIT University, Melbourne, Australia

E-mail: behrooz.ghasemishabankareh@rmit.edu.au

E-mail: melih.ozlen@rmit.edu.au

E-mail: xiaodong.li@rmit.edu.au

K. Deb

Department of Electrical and Computer Engineering, Michigan State University, East Lansing, MI 48824 USA

E-mail: kdeb@egr.msu.edu 


\section{Introduction}

Network models are widely used in practice for solving difficult real-world problems, including a wide range of network optimisation problems such as the shortest path problem, the assignment problem, the maximum flow problem, the minimum cost flow problem (MCFP), the spanning tree problem, etc. Among these, MCFP is one of the fundamental network optimisation problems with many practical applications, e.g., supply chain, logistics, transportation, and production planning [1]. Since the shortest path problem and the maximum cost flow problem are special cases of MCFPs, in this study we consider MCFP as a generic type of network flow models.

The complexity of MCFP highly depends on the shape of its chosen cost function, which computes how much commodities can be sent through the network. An MCFP with a linear cost function is polynomial solvable $[1,2]$. However, the linear cost function may not be able to adequately express the actual cost in a practical situation [3]. For instance, in cargo transportation, factors such as the amount of transportation and transport distance affect the transportation cost function. As a result, the transportation cost may decrease while the amount of cargo increases due to the economy of scale [3]. This scenario shows that the linear and convex cost functions may not be adequate in modelling the real-world scenarios of MCFPs. In contrast, nonlinear nonconvex cost functions that do not make this assumption should represent better the real-world characteristics of the network flow problems $[4,5]$.

A large-scale non-convex MCFP is NP-hard and often considered a challenging problem to be solved in a short period of time, since there are numerous extreme points in a solution set [6,7]. MCFP using a concave cost function is known to be NP-hard [7], where complexity arises from the fact that minimising the concave cost function over a convex feasible region does not guarantee that the global optimum will be found [8].

A single-source uncapacitated MCFP is a special type of MCFPs which has been studied in the past decade. Both exact and approximation methods exist for solving concave MCFPs, among which the branch-and-bound technique is one of the most popular methods for solving single-source uncapacitated MCFPs. For instance, constraint programming and linear programming methods were hybridised with the branch-and-bound method to solve fix-charged MCFP [9]. This hybrid method is twice faster than a commercial integer programming codes. A branch-and-cut algorithm is proposed to solve a single commodity uncapacitated MCFP [10] which consisted of the Steiner tree problem, uncapacitated lot-sizing problems, and the fixed-charge transportation problems as special cases. Other branch-and-bound methods for solving the MCFP can be found in the following works [11-13].

Dynamic programming is another popular mathematical approach for solving MCFPs. Fontes et al. [15] proposed a dynamic programming algorithm to optimally solve single-source uncapacitated MCFPs with linear and concave cost functions, whereas Burkard et al. [14] applied a dynamic programming approach to solve a single-source uncapacitated MCFP using a linear approxi- 
mation of the concave cost function. Furthermore, Erickson et al. [16] proposed a dynamic programming approach called send-and-split method to solve concave MCFPs with single-source and uncapacitated arcs. Kovacs [17] presented an extensive survey on various mathematical programming techniques applied for solving MCFPs.

In addition, many attempts have been made to solve concave single-source uncapacitated MCFPs using metaheuristic methods such as ant colony optimisation (ACO) and GA. Monteiro et al. [8] proposed a hybrid method combining ACO and local search to solve single-source uncapacitated MCFPs. They also carried out a sensitivity study on the parameters used in the ACO algorithm. Other ACO based algorithms for solving the same problems were presented in $[3,18]$. A hybrid method combining GA with local search was also proposed to solve single-source uncapacitated MCFPs and the results were compared with the dynamic programming approach and the upper bound obtained by a local search method [19]. All the aforementioned methods were able to solve the uncapacitated network instances, with the largest network instance considered being networks with 50 nodes.

Literature review suggests only a few limited studies can be found on network flow optimisation using nonlinear non-convex cost functions $[5,20,21]$ mostly focusing on small-sized problems. For example, a nonlinear non-convex transportation problem was solved using an GA [22], and by two exact methods $[23,24]$.

In this paper, we propose a hybrid GA with local search (namely GALS) method to solve the nonlinear non-convex single-source single-sink MCFP. A key novelty of our proposed method is that we use GA to evolve the representation scheme and then local search to refine the searching capability of GALS. Since many real-world MCFPs consist of large-sized networks, this paper shows that GALS is able to handle large-scale MCFPs more effectively. We evaluate our proposed method on a set of 45 network instances, and compare the results with that of a state-of-the-art mathematical solve package, as well as a standard GA. Our results suggest the superiority of GALS over the mathematical solver and the standard GA in solving large-scale MCFPs.

The rest of the paper is structured as follows: the problem definition is presented in Section 2 and the proposed GALS is described in Section 3. Section 4 presents the problem instances and experimental results. Finally the conclusion and future research directions are provided in Section 5.

\section{Problem definition}

Let $G(\mathcal{N}, A)$ be a directed network with $\mathcal{N}=1, \ldots, n$ nodes and a set of $m$ directed $\operatorname{arcs} A$. The upper and lower bounds of flow on each arc $(i, j)$ are denoted by $u_{i, j}$ and $l_{i, j}$ respectively. Instead of a linear or convex cost function, a nonlinear non-convex cost function $f$ is considered in this paper 
and assigned to each $\operatorname{arc}^{1} . q$ represents the supply/demand in the source/sink nodes respectively. $x_{i j}$ is an integer number that denotes the amount of flow through an arc $(i, j)$. Generally in MCFP, we aim to send flows throughout the network to satisfy all demands by minimising the total cost (i.e., the objective function value). Fig. 1 provides a single-source single-sink MCFP example with $n=5$ nodes and $m=7$ arcs. In this example, the aim is to find a flow which satisfies all demands in node 5 ( $\operatorname{sink}$ ) by sending all supplies from node 1 (source) in order to minimise the total cost through the network. The total cost of the single-source single-sink MCFP can be minimised according to the following [1]:

$$
\begin{gathered}
\text { Minimise }: Z(\mathbf{x})=\sum_{i=1}^{n} \sum_{j=1}^{n} f\left(x_{i j}\right), \\
\text { s.t. } \quad \sum_{j=1}^{n} x_{i j}-\sum_{k=1}^{n} x_{k i}= \begin{cases}q, & \text { if } i=1 \\
0, & \text { if } i=(2,3, \ldots, n-1) \\
-q, & \text { if } i=n\end{cases} \\
l_{i j} \leq x_{i j} \leq u_{i j}, \quad(i, j=1, \ldots, n), \\
x_{i j} \in \mathbb{Z}, \quad(i, j=1, \ldots, n),
\end{gathered}
$$

where the cost function in Eq. (1) minimises the total cost within the network. Eq. (2) is the flow balance constraint in which the difference between the first term (total outflow) and the second term (total inflow) is equal to $q$ and $-q$ for source and sink nodes respectively, and is equal to 0 otherwise. Eq. (3) states that the flow on each arc should be within the lower and upper bounds, and finally Eq. (4) ensures that all flow values are integer. Several assumptions of the network we have here include: 1) The network is directed; 2) the network does not contain two or more arcs with the same tail and head nodes; 3) the supply and demand for all nodes except source and sink nodes are equal to 0; 4) the lower bound for each arc $\left(l_{i j}\right)$ has a value of $\left.0 ; 5\right)$ there are no negative cycles in the network; 6$)$ the cost function on each arc $\left(f\left(x_{i j}\right)\right)$ is a nonlinear non-convex function, rather than a linear or convex one.

\section{The proposed GA method}

In this paper, we will demonstrate how a hybrid GA with local search can be applied for solving the MCFP. GA is a stochastic search algorithm inspired by natural selection and genetics [25]. Basically, GA has five main components: representation, a process to create new solutions, evaluation of fitness, genetic operators and parameters [26]. Obviously representation plays a key role in solving many optimization problems (e.g., MCFP), before the GA search can be carried out. In the following section, we will first describe issues associated with the commonly-used priority-based representation scheme, and then we present the proposed GALS for solving MCFPs.

\footnotetext{
1 Some example formulations of nonlinear non-convex cost functions for MCFPs are presented in Section 4.
} 


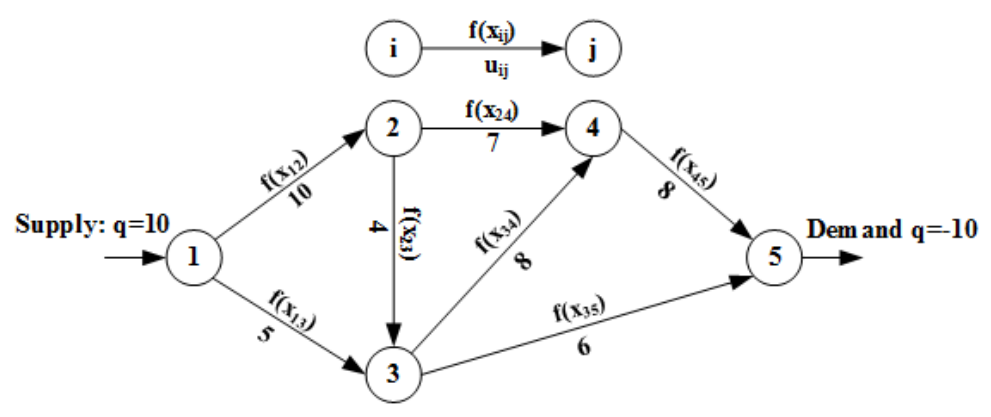

Fig. 1: A single-source single-sink MCFP example.

3.1 Issues with priority-based representation

Priority-based representation has been widely adopted in solving project scheduling, shortest path and network design problems [27-29]. It is one of the most popular approaches to represent an MCFP [30]. In a priority-based representation scheme for MCFPs, the number of genes is equal to the number of nodes $(n)$, and the allele (i.e., the possible value each gene can take) is created randomly between 1 and the number of nodes $(n)$ (Fig. 2a).

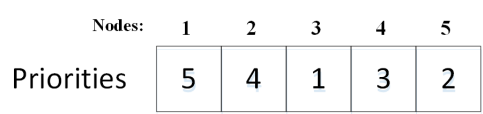

(a) A chromosome for the prioritybased encoding method.

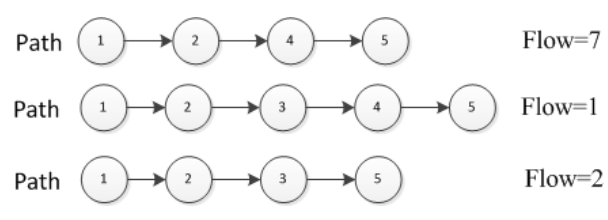

(b) Paths produced from the priority chromosome shown in (a).

Fig. 2: A chromosome and its MCFP solution for the network in Fig. 1.

From the priority chromosome, several paths can be constructed in order to satisfy the demand of MCFP presented in Fig. 1. These paths constitutes an appropriate MCFP solution. As shown in Fig. 2b, we can construct several paths starting from node 1 , ending in node 5 . For each path, starting from node 1 , we select the successor node with a higher priority. For example, the successors for node 1 are nodes 2 and 3 . Based on the priority chromosome, the priorities of nodes 2 and 3 are values of 4 and 1 respectively. Hence node 2 is chosen. After node 2, the successor nodes are nodes 3 and 4 . Since the priority of node 3 (1) is smaller than that of node 4 (3), node 4 is chosen as the destination and from node 4 the only possible successor node is node 5. Finally, the completed path is $1 \rightarrow 2 \rightarrow 4 \rightarrow 5$. The possible flow on this path is equal to the minimum of capacities on the arcs of the path and the supply/demand (Flow $=\min \{10,7,8, \mathrm{q}=10\}=7)$. At this point, we need to update the capacities 
on the arcs, supply, and demand. If the demand in node 5 is not satisfied, then the second path is constructed, similarly as the previous path. This process continues until the demand is satisfied. Fig. $2 \mathrm{~b}$ shows the 3 paths produced from the above process.

The priority-based representation method is unable to encode all the possible solutions in the feasible search space, as shown in Fig. 3a. For example, Fig. 3b shows an MCFP instance, where the priority-based representation method fails to realise.

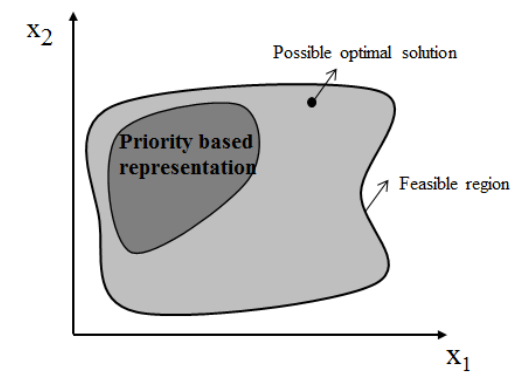

(a) The priority-based encoding only represents partially the feasible space.

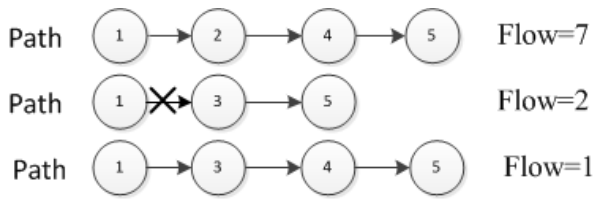

(b) A feasible MCFP solution that prioritybased encoding fails to represent.

Fig. 3: Disadvantages of the priority-based encoding method.

\subsection{Improved priority-based encoding}

To address the above issue, we propose an improved priority-based encoding (iPE) method here. In iPE, the locus, (i.e., the position (or index) of the gene) and allele of the main chromosome are identical to the priority-based encoding method. The main difference is that after the first path is constructed, from the second path onwards, instead of using the same chromosome, two genes of the main chromosome are randomly swapped and the new path is then constructed based on this new chromosome. In other words, it is now possible to generate MCFP solutions based on newly produced representation instances, rather than one fixed presentation instance. Algorithm 1 shows the procedure of iPE in detail. By using the swapping technique in the main chromosome, the priority-based method is redeemed and it is now possible to represent more feasible solutions using this iPE method.

3.3 GALS for solving single-source single-sink MCFPs

Building on iPE method, this section proposes GALS for solving large-scale MCFPs with nonlinear non-convex cost functions, where iPE is used to per- 


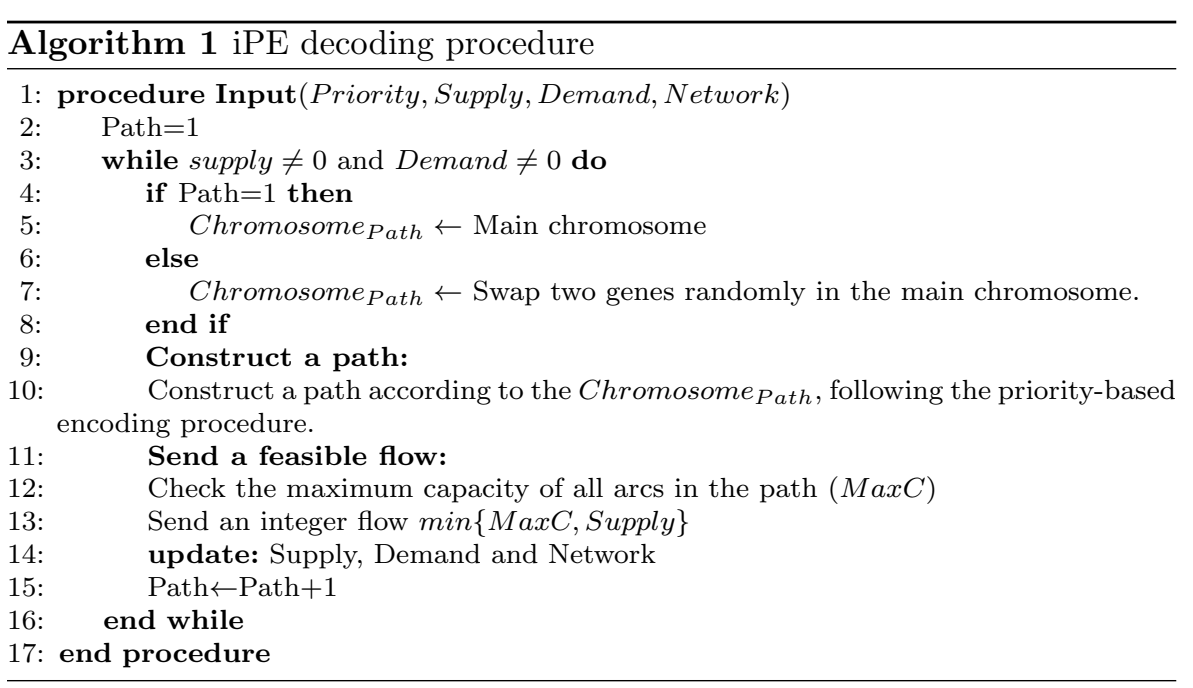

form local search to further enhance the searching capability of GALS, which involves the following procedure:

Initialisation: A population of $n_{\text {pop }}$ individuals (chromosomes) is first randomly generated (according to Subsection 3.1).

Crossover and mutation: Crossover and mutation operators are then applied to create a new offspring population. For each newly generated offspring, two parents are first randomly selected and a weight mapping crossover (WMX) is applied [30]. Subsequently an inversion mutation operator is applied [30].

Solution decoding: To counteract the limitations of the priority-based representation, the iPE decoding procedure (as shown in Algorithm 1) is performed $N$ times for each chromosome in the population. As shown in Fig. 4, after performing the decoding, $N$ solutions are obtained.

Evaluation and local search: The $N$ number of MCFP solutions generated from the previous decoding step (with respect to each chromosome) are evaluated using Eq. (1). A local search is carried out by selecting the decoded solution with the smallest cost, among all $N$ solutions.

Population update: After evaluating all individuals in the population, the tournament selection procedure (with a tournament size of 2) is applied to select the fitter individuals for the next generation.

Termination criteria: The above process continues until a stopping criterion is met, which is either 1 ) no further fitness value improvement in the best individual of the population for $\beta$ successive iterations; or 2 ) the maximum number of function evaluations (NFEs) is reached. 


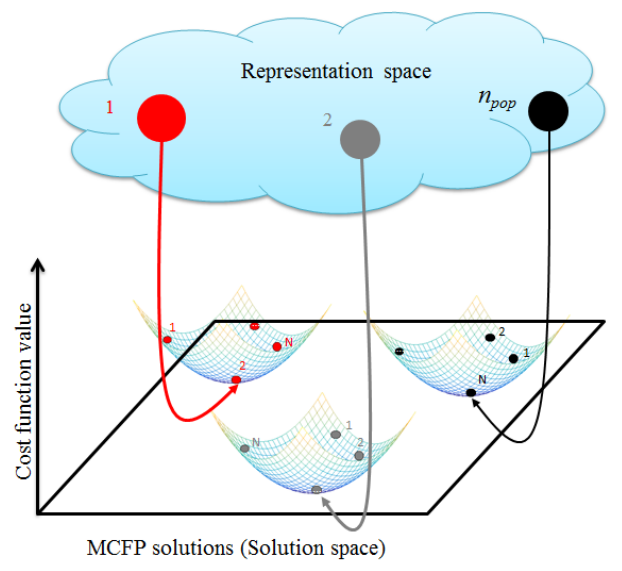

Fig. 4: The local search procedure for GALS (adapted from [36]).

\section{Test problems}

We focus on evaluating GALS on the single-source single-sink MCFP using nonlinear and non-convex cost functions. Several different types of nonlinear non-convex functions were suggested by Michalewicz [22] on the transportation problems. We select the following nonlinear non-convex cost functions (as shown in Fig. 5) for our study as they are considered to be more practical and challenging than others [22-24]:

$$
\begin{aligned}
f_{1}\left(x_{i j}\right)= & \arctan \left(P A\left(x_{i j}-S\right)\right) / \pi+0.5+ \\
& \arctan \left(P A\left(x_{i j}-2 S\right)\right) / \pi+0.5+ \\
& \arctan \left(P A\left(x_{i j}-3 S\right)\right) / \pi+0.5+ \\
& \arctan \left(P A\left(x_{i j}-4 S\right)\right) / \pi+0.5+ \\
& \arctan \left(P A\left(x_{i j}-5 S\right)\right) / \pi+0.5, \\
f_{2}\left(x_{i j}\right)= & 100 \times\left(x_{i j}\left(\sin \left(\frac{5 \pi x_{i j}^{w}}{4 S}\right)+1.3\right)\right),
\end{aligned}
$$

where the values of $P A$ is set to 1000 and $S$ is set to 2 for $f_{1}$, and 5 for $f_{2}$, respectively [23]. To examine the robustness of the proposed algorithm, the parameter $w$ in the cost function $f_{2}$ is set to 1,2 and 3 , to generate $f_{2 a}, f_{2 b}$ and $f_{2 c}$ functions, respectively. For our evaluation purpose, random networks are created with different sizes from 5 to 100 nodes and with a random number of arcs (decision variables) from 7 to approximately 2500. These network instances are categorised in small, medium-sized (5 to 40 nodes), and largesized problems (60 to 100 nodes). All these network instances are used for evaluating our proposed algorithm. Our results are compared with those of the commercial mathematical programming solver namely LindoGlobal [35] and the standard GA. 


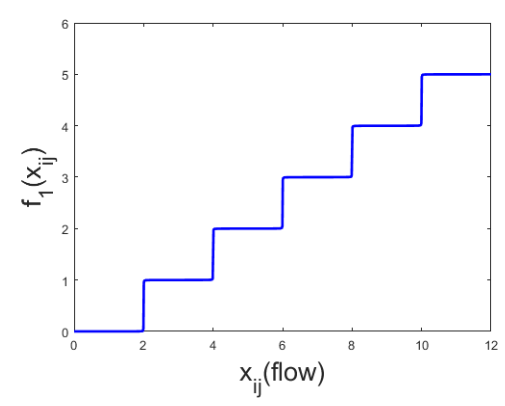

(a) $f_{1}$

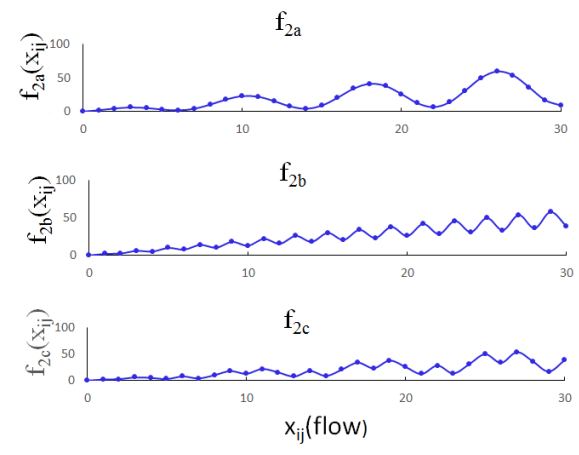

(b) $f_{2}$

Fig. 5: The shape of the cost functions are presented in Eqs. (5) and (6).

4.1 Mathematical solvers

Although exact and heuristic methods exist for solving an MINLP where the objective and constraints are convex, in practice most of the functions are non-convex, which makes the problem extremely difficult to solve [31]. The relaxation of a non-convex MINLP (to make it convex) is itself a global optimisation problem, and it is likely to be NP-hard [32,33]. Some representative algorithms for solving non-convex MINLPs include spatial branch-and-bound, branch-and-reduce and $\alpha$ branch-and-bound [31]. Based on the aforementioned algorithms, some commercial and open source solvers have been developed for solving non-convex MINLPs, such as CPLEX, Baron, Couenne and LindoGlobal [31].

Nevertheless, these mathematical solver packages have many limitations. For instance, CPLEX is probably one of the most powerful solvers, but it can only handle mixed-integer quadratic programs under certain conditions for constraints and objective functions. Clearly, CPLEX is unable to handle other types of nonlinear functions. The solvers that are able to solve the general nonconvex MINLPs include BARON, LindoGlobal, Couenne [31]. BARON [34] is unable to handle trigonometric functions $\sin (x), \cos (x)$, and Couenne is unable to handle the arctangent functions. Among these solvers, LindoGlobal is the only solver that can handle different types of nonlinear functions [35]. Hence in this paper we compare our proposed method GALS with LindoGlobal as well as the standard GA. Unlike GAs, the capability and performance of the mathematical solvers are highly dependent on the shapes of the cost functions adopted in the non-convex MINLPs. 
4.2 Numerical results

Our proposed method GALS and the standard GA are implemented in MATLAB on a PC with Intel(R) Core(TM) i7-6500U $2.50 \mathrm{GHz}$ processor with 8 GB RAM and run 30 times for each problem instance using cost function $f_{1}$, $f_{2 a}, f_{2 b}$ and $f_{2 c}$. The computational results for GALS, LindoGlobal and the standard GA, are presented in Tables 1 to 4 .

Parameter settings of GALS are as follows: maximum number of iteration $\left(I t_{\max }=100\right)$, population size $\left(n_{p o p}=50\right)$, number of local search for each individual $(N=20)$, crossover rate $(\mathrm{Pc}=0.95)$, mutation rate $(\mathrm{Pm}=0.3)$. The parameter settings for the standard GA method are as follows: $I t_{\max }=500$, $n_{\text {pop }}=200, \mathrm{Pc}=0.95, \mathrm{Pm}=0.3$. For both methods, the NFEs and $\beta$ are set to 150,000 and 20 respectively.

In Tables 1 to 4, for the non-deterministic methods (GALS and standard GA), b, std and t denote the best, standard deviation of the results and the average of running time in seconds respectively, and the mean represents the average of objective function values over 30 runs and finally $h$ denotes the result of pairwise $t$-test. For LindoGlobal, the objective function value is recorded in "Obj1" column after t1 seconds and the LindoGlobal keeps running for t2 seconds and the final objective function value is recorded in "Obj2" column. All computational times are reported in seconds.

As shown in Tables 1 to 4, the highest average time for GALS is 286 seconds. To make the results more comparable, we record the objective value found by LindoGlobal at 300s, as reported in "Obj1" column (note that "-" in "t1" and "Obj1" indicates that the results are identical to those in "t2" and "Obj2", in which case LindoGlobal found the global optimum before reaching $300 \mathrm{~s})$. We also allow LindoGlobal to keep running until either a global optima is found or the termination time $(3,600 \mathrm{~s})$ is reached, and the results are reported in the "Obj2" column. As can be seen in Tables 1 to 4, LindoGlobal can only find global optima for small instances ( 5 and 10 nodes).

In order to compare the performance of GALS and the standard GA, the $t$ test with the significance level of 0.05 is performed. If GALS is equal, superior or inferior to the standard GA, then $h$ is set to 0,1 and -1 respectively. We also compare the performance of GALS with LindoGlobal by performing a onesample $t$-test with the significance level set to 0.05 and either of the methods has better or equal performance than that of the other is highlighted in bold.

Fig. 6 summarises the results of GALS, the standard GA and LindoGlobal for solving all instances of different sizes using nonlinear non-convex cost functions $f_{1}, f_{2 a}, f_{2 b}$ and $f_{2 c}$. As shown in Table 1 and for all 45 problem instances, GALS is superior $39(87 \%)$ times, equal 6 times (13\%), and inferior 0 times, when compared with the standard GA. When comparing the mean values of GALS with LindoGlobal on all 45 problem instances (cost function $f_{1}$, Table 1 ), GALS is superior 30 times (67\%), equal 11 times (24\%) and inferior 4 times $(9 \%)$. It is noticeable that LindoGlobal cannot find any feasible solutions on all the large-sized problems in 3,600 seconds using cost function $f_{1}$. 
Table 1: Results of GALS, GA, and LindoGlobal using cost function $f_{1}$.

\begin{tabular}{|c|c|c|c|c|c|c|c|c|c|c|c|c|c|c|c|}
\hline \multicolumn{16}{|c|}{ Small and medium-sized instances } \\
\hline No. & Nodes & Arcs & & & GALS & & & & GA & & $h$ & & LIND & OGloba & \\
\hline & \multirow{6}{*}{5} & & $t$ & $\mathrm{~b}$ & mean & std & $\mathrm{t}_{\mathrm{t}}$ & $\mathrm{b}$ & mean & std & & t1 & Obj1 & $\mathrm{t}_{2}$ & $\mathrm{Obj} 2$ \\
\hline 1 & & 7 & 16 & 5.0021 & 5.0021 & $9.11 \mathrm{E}-16$ & 32 & 5.0021 & 5.0021 & $6.13 \mathrm{E}-08$ & 0 & & & 2 & 5.0021 \\
\hline 2 & & 7 & 17 & 5.0021 & 5.0021 & $9.11 \mathrm{E}-16$ & 34 & 5.0021 & 5.0021 & $4.15 \mathrm{E}-13$ & 0 & - & - & 2 & 5.0021 \\
\hline 3 & & 8 & 16 & 5.0024 & 5.0024 & $0.00 \mathrm{E}+00$ & 33 & 5.0024 & 5.0024 & $3.07 \mathrm{E}-08$ & 0 & - & - & 1 & 5.0024 \\
\hline 4 & & 10 & 18 & 5.0032 & 5.0032 & $1.82 \mathrm{E}-15$ & 32 & 5.0032 & 5.0032 & $2.73 \mathrm{E}-12$ & 0 & - & - & 1 & 5.0032 \\
\hline 5 & & & 18 & 5.0024 & 5.0024 & $0.00 \mathrm{E}+00$ & 33 & 5.0024 & 5.0024 & $1.36 \mathrm{E}-07$ & 0 & - & - & 1 & 5.0024 \\
\hline 6 & \multirow{5}{*}{10} & 32 & 26 & 10.0107 & 10.0107 & $0.00 \mathrm{E}+00$ & 44 & 10.2021 & 10.2023 & $1.75 \mathrm{E}-06$ & 1 & - & - & 5 & 10.0107 \\
\hline 7 & & 25 & 26 & 10.0081 & 10.0081 & 3.33E-15 & 42 & 10.1461 & 10.1707 & $1.93 \mathrm{E}-02$ & 1 & & & 7 & 10.0081 \\
\hline 8 & & 34 & 29 & 10.0114 & 10.0114 & 4.66E-15 & 42 & 10.2024 & 10.2036 & 8. $32 \mathrm{E}-04$ & 1 & 300 & 10.0114 & 949 & 10.0114 \\
\hline 9 & & 20 & 42 & 10.0063 & 10.0063 & $2.73 \mathrm{E}-15$ & 40 & 10.2103 & 10.2495 & $6.97 \mathrm{E}-03$ & 1 & & & 2 & 10.0063 \\
\hline 10 & & 32 & 40 & 10.0107 & 10.0107 & $0.00 \mathrm{E}+00$ & 46 & 10.2037 & 10.2227 & 3.51E-03 & 1 & 300 & 10.0107 & 3600 & 10.0107 \\
\hline 11 & \multirow{5}{*}{20} & 123 & 64 & 6.5500 & $\begin{array}{c}1.0107 \\
9.2463\end{array}$ & $1.45 \mathrm{E}+00$ & 72 & $\begin{array}{l}1.2003 \\
9.0463\end{array}$ & $\begin{array}{l}1.2221 \\
9.9089\end{array}$ & $\frac{5.01 \mathrm{E}-0.0}{4.18 \mathrm{E}-01}$ & $\frac{1}{0}$ & 300 & $\frac{1.0101}{6.0494}$ & 3600 & $\frac{10.0101}{6.0490}$ \\
\hline 12 & & 142 & 61 & 4.0571 & 4.8542 & 2.76E- 01 & 63 & 7.5546 & 8.0226 & $9.32 \mathrm{E}-01$ & 1 & 300 & 4.0572 & 3600 & 4.0569 \\
\hline 13 & & 88 & 48 & 10.0310 & 10.0310 & $0.00 \mathrm{E}+00$ & 59 & 12.0475 & 12.0475 & 3.47E-06 & 1 & 300 & 9.5374 & 3600 & 9.0370 \\
\hline 14 & & 133 & 68 & 13.5528 & 15.3900 & $1.23 \mathrm{E}+00$ & 70 & 15.5511 & 16.6254 & $8.64 \mathrm{E}-01$ & 1 & 300 & 15.0554 & 3600 & 15.0553 \\
\hline 15 & & 117 & 63 & 14.5461 & 16.1869 & $9.74 \mathrm{E}-01$ & 64 & 16.5434 & 17.0505 & $7.27 \mathrm{E}-01$ & 1 & 300 & 19.5448 & 3600 & 18.5456 \\
\hline 16 & \multirow{5}{*}{40} & 363 & 66 & 10.1311 & 10.1311 & $0.00 \mathrm{E}+00$ & 81 & 11.2196 & 12.4407 & $1.69 \mathrm{E}-01$ & 1 & 300 & $\mathrm{NF}$ & 3600 & 7.6402 \\
\hline 17 & & 295 & 64 & 10.1063 & 10.1063 & $3.50 \mathrm{E}$ & 85 & 12.2687 & 13.2361 & $2.13 \mathrm{E}-01$ & 1 & 300 & $\mathrm{NF}$ & 3600 & 14.6145 \\
\hline 18 & & 320 & 66 & 10.1154 & 10.1154 & $2.25 \mathrm{E}-14$ & 93 & 11.1039 & 13.0880 & $1.99 \mathrm{E}+00$ & 1 & 300 & NF & 3600 & 12.6241 \\
\hline 19 & & 343 & 69 & 10.1238 & 10.1238 & $4.45 \mathrm{E}-15$ & 95 & 13.1794 & $\begin{array}{l}14.0000 \\
14.0112\end{array}$ & $6.23 \mathrm{E}-01$ & 1 & 300 & NF & 3600 & 13.1331 \\
\hline 20 & & 294 & 65 & 10.1060 & 10.1060 & $0.00 \mathrm{E}+00$ & 80 & 12.1860 & 13.0349 & $3.83 \mathrm{E}-01$ & 1 & 300 & $\mathrm{NF}$ & 3600 & $\mathrm{NF}$ \\
\hline & \multicolumn{15}{|c|}{ gge-sized instances } \\
\hline 21 & \multirow{5}{*}{60} & 844 & 121 & 3.8220 & 7.5678 & $2.67 \mathrm{E}+00$ & $\frac{149}{149}$ & 9.3142 & 13.4360 & $2.83 \mathrm{E}+00$ & & 300 & $\mathrm{NF}$ & 3600 & $\mathrm{NF}$ \\
\hline 22 & & 905 & 127 & 6.8411 & 10.0384 & $2.10 \mathrm{E}+00$ & 167 & 13.3323 & 15.1815 & $1.59 \mathrm{E}+00$ & 1 & 300 & NF & 3600 & NF \\
\hline & & 798 & 111 & 6.3024 & 9.7742 & $2.34 \mathrm{E}+00$ & 124 & 11.2962 & 14.1178 & $1.65 \mathrm{E}+00$ & 1 & 300 & $\mathrm{NF}$ & 3600 & NF \\
\hline 24 & & 912 & 124 & 4.8475 & 9.0931 & $2.35 \mathrm{E}+00$ & 203 & 12.8363 & 15.5336 & $1.54 \mathrm{E}+00$ & 1 & 300 & NF & 3600 & NF \\
\hline 25 & & 870 & 127 & 3.3310 & 6.9530 & $2.27 \mathrm{E}+00$ & 188 & 10.3215 & 12.5202 & $1.25 \mathrm{E}+00$ & 1 & 300 & $\mathrm{NF}$ & 3600 & $\mathrm{NF}$ \\
\hline 26 & \multirow{5}{*}{70} & 1176 & 138 & 4.4403 & $\begin{array}{l}7.35390 \\
7.3391\end{array}$ & $\frac{2.21 \mathrm{E}+00}{2.25 \mathrm{E}+00}$ & 160 & 10.4329 & 13.7305 & $\frac{1.205 \mathrm{E}+00}{2.05+00}$ & 1 & 300 & $\mathrm{NF}$ & 3600 & NF \\
\hline 27 & & 1163 & 146 & 3.4391 & 6.9352 & $1.86 \mathrm{E}+00$ & 169 & 10.4280 & 12.5263 & $2.24 \mathrm{E}+00$ & 1 & 300 & $\mathrm{NF}$ & 3600 & NF \\
\hline 28 & & 1231 & 152 & 1.4621 & 4.8605 & $1.36 \mathrm{E}+00$ & 189 & 10.4547 & 12.7022 & $1.14 \mathrm{E}+00$ & 1 & 300 & $\mathrm{NF}$ & 3600 & NF \\
\hline 29 & & 991 & 148 & 4.3745 & 8.3473 & $2.23 \mathrm{E}+00$ & 174 & 9.8679 & 13.5143 & $1.72 \mathrm{E}+00$ & 1 & 300 & $\mathrm{NF}$ & 3600 & NF \\
\hline 30 & & 1252 & 133 & 3.4695 & 6.6675 & $2.29 \mathrm{E}+00$ & 187 & 10.4619 & 14.4336 & $2.79 \mathrm{E}+00$ & 1 & 300 & NF & 3600 & NF \\
\hline 31 & \multirow{5}{*}{80} & 1718 & 151 & 3.1368 & 5.6617 & $1.83 \mathrm{E}+00$ & 193 & 8.1334 & 11.4305 & $2.25 \mathrm{E}+00$ & 1 & 300 & $\mathrm{NF}$ & 36 & NF \\
\hline 32 & & 1812 & 142 & 3.1710 & 5.17 & $1.53 \mathrm{E}+00$ & 144 & 9.16 & 11.7380 & $2.52 \mathrm{E}+00$ & 1 & 30 & $\mathrm{NF}$ & 3600 & NF \\
\hline 33 & & 1513 & 133 & 3.0649 & 6.3 & $2.67 \mathrm{E}+00$ & 194 & 10.0568 & 13.7 & -00 & 1 & 300 & $\mathrm{NF}$ & 3600 & $\mathrm{NF}$ \\
\hline 34 & & 1880 & 157 & 3.1 & 4.7 & & $\begin{array}{l}194 \\
193\end{array}$ & 9.68 & & & 1 & 300 & $\mathrm{NI}$ & 36 & NF \\
\hline $\begin{array}{l}34 \\
35\end{array}$ & & $\begin{array}{l}1800 \\
1619\end{array}$ & $\begin{array}{l}142 \\
142\end{array}$ & $\begin{array}{l}3.19037 \\
1.6037\end{array}$ & $\begin{array}{l}4.1 \\
4.0\end{array}$ & $1.24 \mathrm{E}+00$ & $\begin{array}{l}190 \\
201\end{array}$ & $\begin{array}{l}9.0092 \\
10.0940\end{array}$ & $\begin{array}{l}12.4894 \\
12.5183\end{array}$ & $\begin{array}{l}2.00 \mathrm{E}+00 \\
2.09 \mathrm{E}+00\end{array}$ & 1 & 300 & NF & $\begin{array}{l}3000 \\
3600\end{array}$ & NF \\
\hline 36 & \multirow{5}{*}{90} & 1893 & 165 & 2.7037 & 6.3511 & $2.66 \mathrm{E}+00$ & 209 & 10.1936 & 13.3174 & $2.47 \mathrm{E}+00$ & 1 & 300 & NF & 3600 & NF \\
\hline 37 & & 2013 & 202 & 3.2470 & 5.8454 & $2.34 \mathrm{E}+00$ & 232 & 11.2367 & 13.5627 & $1.92 \mathrm{E}+00$ & 1 & 300 & $\mathrm{NF}$ & 3600 & NF \\
\hline 38 & & 2185 & 164 & 2.8087 & 5.8079 & $2.40 \mathrm{E}+00$ & 219 & 9.3009 & 11.6497 & $1.25 \mathrm{E}+00$ & 1 & 300 & NF & 3600 & NF \\
\hline 39 & & 1944 & 159 & 5.2195 & 10.0152 & $3.22 \mathrm{E}+00$ & 210 & 12.2108 & 14.0598 & $904 \mathrm{E}-01$ & 1 & 300 & $\mathrm{NF}$ & 3600 & $\mathrm{NF}$ \\
\hline 40 & & 2013 & 171 & 2.7462 & 4.9947 & $2.06 \mathrm{E}+00$ & 277 & 8.7389 & 12.9856 & $2.33 \mathrm{E}+00$ & 1 & 300 & NF & 3600 & NF \\
\hline 41 & \multirow{5}{*}{100} & & 239 & 1.4 & & & $\frac{21}{28}$ & & & & 1 & (2000 & Nit & & \\
\hline 42 & & 2512 & 283 & & & & 292 & $9.4 \quad \longrightarrow$ & 14.0 & 00 & 1 & 300 & $\mathrm{NI}$ & 3600 & NF \\
\hline 43 & & 2437 & 228 & 2.4001 & 4.5490 & $1.20 \mathrm{E}+00$ & 255 & 7.8930 & 10.6174 & $1.63 \mathrm{E}+00$ & 1 & 300 & $\mathrm{NF}$ & 3600 & NF \\
\hline 44 & & 2370 & 279 & 2.3758 & 4.7743 & $2.03 E+00$ & 310 & 9.8683 & 11.0182 & $5.40 \mathrm{E}-01$ & 1 & 300 & NF & 3600 & NF \\
\hline 45 & & 2503 & 265 & 2.9247 & 8.0457 & $3.32 \mathrm{E}+00$ & 267 & 10.9191 & 13.3393 & $1.42 \mathrm{E}+00$ & 1 & 300 & $\mathrm{NF}$ & 3600 & NF \\
\hline
\end{tabular}

To examine if GALS is robust to the different shapes of a cost function, we use cost function $f_{2}$ (Eq. 6), choosing three different values for the parameter $w$ in Eq. 6. As shown in Fig. 5b, by increasing the parameter $w$ from 1, to 2 and 3 , the number of peaks and valleys (local optima) are increased gradually in functions $f_{2 b}, f_{2 c}$. Dealing with these cost functions will be a challenging task. A robust optimisation algorithm should be able to handle this sort of highly non-convex shaped cost functions, without degrading their performances.

As can be seen in Table 2, although LindoGlobal outperformed GALS on some small and medium size problem instances, GALS achieved significantly better performances than those of LindoGlobal on large-sized problems. Additionally, in Tables 3 and 4, GALS significantly outperforms LindoGlobal on all large-sized instances and LindoGlobal has increasing difficulty in finding feasible solutions on instances with $70,80,90$ and 100 nodes using cost function $f_{2 b}$ as well as on instances with 80,90 and 100 nodes using cost function $f_{2 c}$ (except instances No.35,39,40). It is evident that the mathematical solver is sensitive to the non-convex shapes introduced in the cost functions $f_{2 a}, f_{2 b}$, and $f_{2 c}$. In contrast, GALS performance is much more robust with respect to 
Table 2: Results of GALS, GA, and LindoGlobal using cost function $f_{2 a}$.

\begin{tabular}{|c|c|c|c|c|c|c|c|c|c|c|c|c|c|c|c|}
\hline \multicolumn{16}{|c|}{ Small and medium-sized instances } \\
\hline No. & Nodes & Arcs & & & GALS & & & & & & $h$ & & LIND & Globa & \\
\hline & \multirow{5}{*}{5} & & $\mathrm{t}$ & $\mathrm{b}$ & mean & std & $\mathrm{t}$ & $\mathrm{b}$ & mean & $\frac{s t d}{309515}$ & & $\mathrm{t} 1$ & Obj1 & $\mathrm{t} 2$ & $\mathrm{Obj} 2$ \\
\hline $\begin{array}{l}1 \\
2\end{array}$ & & 7 & $\begin{array}{l}18 \\
16\end{array}$ & $\begin{array}{c}9.6000 \\
11.4000\end{array}$ & $\begin{array}{c}9.6000 \\
11.4000\end{array}$ & $\begin{array}{l}2.92 \mathrm{E}-12 \\
5.16 \mathrm{E}-11\end{array}$ & $\begin{array}{l}17 \\
16\end{array}$ & $\begin{array}{l}26.0000 \\
26.0000\end{array}$ & $\begin{array}{c}9.6000 \\
11.4000\end{array}$ & $\begin{array}{l}7.29 \mathrm{E}-15 \\
7.29 \mathrm{E}-15\end{array}$ & $\begin{array}{l}0 \\
0\end{array}$ & - & - & $\begin{array}{l}1 \\
1\end{array}$ & $\begin{array}{r}9.6000 \\
11.4000\end{array}$ \\
\hline 3 & & 8 & 18 & 9.6000 & 9.6000 & $6.21 \mathrm{E}-10$ & 17 & 26.0000 & 9.6000 & $7.29 \mathrm{E}-15$ & 0 & - & - & 1 & 9.6000 \\
\hline 4 & & 10 & 19 & 11.4000 & 11.4000 & $5.24 \mathrm{E}-12$ & 19 & 26.0000 & 11.4000 & $7.29 \mathrm{E}-15$ & 0 & - & - & 2 & 11.4000 \\
\hline 5 & & 8 & 17 & 9.6000 & 9.6000 & $4.86 \mathrm{E}-14$ & 17 & 26.0000 & 9.6000 & $7.29 \mathrm{E}-15$ & 0 & - & 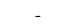 & 1 & 9.6000 \\
\hline 0 & \multirow{5}{*}{10} & 32 & 23 & 32.4853 & 32.6610 & $4.29 \mathrm{E}-01$ & 27 & 33.6569 & 33.6569 & $0.00 \mathrm{E}+00$ & +5 & & & 5 & 21.0000 \\
\hline 7 & & 25 & 23 & 29.4355 & 29.4355 & $3.65 \mathrm{E}-15$ & 23 & 30.6000 & 30.8828 & $6.91 \mathrm{E}-01$ & 1 & - & & 42 & 29.4284 \\
\hline 8 & & 34 & 25 & 31.8142 & 32.1827 & $7.56 \mathrm{E}-01$ & 27 & 33.6569 & 33.6569 & $0.00 \mathrm{E}+00$ & 1 & - & & 50 & 21.0000 \\
\hline 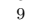 & & 20 & 30 & 35.6640 & 36.1 & $6.09 \mathrm{E}-01$ & 29 & 45.5431 & 45.9446 & $8.24 \mathrm{E}-01$ & 1 & - & & 10 & 32.0213 \\
\hline 10 & & 32 & 27 & 33.2000 & 33.5 & $.32 \mathrm{E}$ & 26 & 34.7147 & 34 & $4.29 \mathrm{E}-01$ & & - & & 295 & 32.0213 \\
\hline 11 & \multirow{5}{*}{20} & 123 & 65 & 32.4853 & 32.4853 & $7.29 \mathrm{E}-15$ & 66 & 32.4853 & 34.6143 & $.57 \mathrm{E}+00$ & 1 & 300 & 32.4853 & 3600 & 32.4853 \\
\hline 12 & & 142 & 75 & 32.4853 & 32.4853 & $4.61 \mathrm{E}-15$ & 78 & 32.4853 & 32.4853 & $3.65 \mathrm{E}-15$ & 0 & 300 & 30.8569 & 3600 & 30.8569 \\
\hline 13 & & 88 & 70 & 35.6640 & 37.6254 & $2.58 \mathrm{E}+00$ & 71 & 38.2569 & 40.7929 & $3.36 \mathrm{E}+00$ & 1 & 300 & 28.4938 & 3600 & 28.2000 \\
\hline 14 & & 133 & 77 & 75.7218 & 76.6429 & $1.06 \mathrm{E}+00$ & 79 & 75.8934 & 77.6869 & $8.29 \mathrm{E}-01$ & 1 & 300 & 65.9208 & 3600 & 65.3279 \\
\hline 15 & & 117 & 70 & 75.0132 & 76.2276 & $1.14 \mathrm{E}+00$ & 74 & 75.0132 & 78.0872 & $2.14 \mathrm{E}+00$ & 1 & 300 & 71.9635 & 3600 & 67.1635 \\
\hline 16 & \multirow{5}{*}{40} & 363 & 83 & 18.0000 & 18.0000 & $0.00 \mathrm{E}+00$ & 94 & 18.0000 & 18.0000 & $0.00 \mathrm{E}+00$ & 0 & 300 & 89.6061 & 3600 & 18.0000 \\
\hline 17 & & 295 & 81 & 18.0000 & 18.0000 & $0.00 \mathrm{E}+00$ & 82 & 18.0000 & 18.0000 & $0.00 \mathrm{E}+00$ & 0 & 300 & 95.6430 & 3600 & 18.0000 \\
\hline 18 & & 320 & 85 & 18.0000 & 18.0000 & $0.00 \mathrm{E}+00$ & 80 & 18.0000 & 18.0000 & $0.00 \mathrm{E}+00$ & 0 & 300 & 104.5940 & 3600 & 18.0000 \\
\hline 19 & & 343 & 82 & 18.0000 & 18.0000 & $0.00 \mathrm{E}+00$ & 77 & 18.0000 & 18.0000 & $0.00 \mathrm{E}+00$ & 0 & 300 & 104.7280 & 3600 & 18.0000 \\
\hline 20 & & 294 & 84 & 18.0000 & 18.0000 & $0.00 \mathrm{E}+00$ & 80 & 18.0000 & 18.0000 & $0.00 \mathrm{E}+00$ & & 300 & 113.4920 & 3600 & 18.0000 \\
\hline \multicolumn{16}{|c|}{ (0) } \\
\hline 21 & \multirow{5}{*}{60} & 844 & 114 & 72.4284 & 74.1 & & 109 & 73.8 & & & & & & & 29 \\
\hline 22 & & 90 & 119 & 72.1563 & 72.1563 & $1.46 \mathrm{E}-14$ & 118 & 73.7990 & 76.9609 & $2.21 \mathrm{E}+00$ & 1 & 30 & NF & 3600 & 72.6274 \\
\hline 23 & & 79 & 102 & 74.9706 & 75.8 & $1.17 \mathrm{E}$ & 04 & & & & 1 & & $\mathrm{NF}$ & & 71.0711 \\
\hline 24 & & 912 & 120 & 74.9706 & 75.1962 & 4.07 & 122 & 74. & 78 & +00 & 1 & 300 & $\mathrm{NF}$ & 3600 & 72.6274 \\
\hline 25 & & 870 & 115 & 73.7990 & 74.2090 & 5.73E-01 & 125 & 73.7990 & 76.9813 & $2.53 \mathrm{E}+00$ & 1 & 300 & $\mathrm{NF}$ & 3600 & 72.6274 \\
\hline 26 & \multirow{5}{*}{70} & 1176 & 140 & 72.6274 & 73.4 & $9.39 \mathrm{E}-01$ & 135 & 73.7 & & 2.0 & 1 & 30 & $\mathrm{NF}$ & 3600 & 70.2426 \\
\hline 27 & & & 134 & & & & 135 & & & & 1 & 30 & $\mathrm{NF}$ & 3600 & 72.6274 \\
\hline 28 & & 1231 & 140 & 72. & 72.6 & 2.9 & 143 & 74. & 77 & 1.2 & 1 & 30 & $\mathrm{NF}$ & 3600 & 73.7990 \\
\hline 29 & & & 125 & 75.8 & & & 129 & & & & 1 & 30 & NF & 3600 & 77.3208 \\
\hline 30 & & 1252 & 135 & 76.1 & & & 133 & & & & 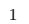 & 30 & $\mathrm{NF}$ & 3600 & 104.5198 \\
\hline 31 & \multirow{5}{*}{8} & & 154 & 74.9 & & 1.52 & 148 & & & & & & & & 99.2061 \\
\hline 32 & & & 15 & & & & 15 & & & & & & & & \\
\hline 33 & & & 13 & & & & 13 & & & & & & & & \\
\hline 34 & & & 16 & & & & 16 & & & & & & & & \\
\hline $\begin{array}{l}54 \\
35\end{array}$ & & 16 & $\begin{array}{l}155 \\
155\end{array}$ & & & & 57 & & & & 1 & & NF & & \\
\hline 36 & \multirow{5}{*}{90} & & 161 & & & & 18 & & & & 1 & & $\mathrm{NF}$ & & \\
\hline 37 & & & 18 & & & & 18 & 77.3 & & & 1 & & $\mathrm{NF}$ & & 78.5 \\
\hline 38 & & & 193 & 72. & & & 187 & & 78 & 2.5 & 1 & 30 & NF & 3600 & 72.7990 \\
\hline 39 & & & 181 & 72.7 & 73.7 & & 184 & 74.4 & & & 1 & & $\mathrm{NF}$ & 3600 & 104.2487 \\
\hline 40 & & 2013 & 193 & 73.7990 & 74.3 & $5.52 \mathrm{E}$ & 195 & 75.5289 & 76.8 & 1.00 & 1 & 300 & NF & 3600 & 68.4061 \\
\hline 41 & \multirow{5}{*}{100} & 2501 & 248 & 78.1492 & 78.6 & 4.191 & 241 & 80.3431 & 82. & $1.85 \mathrm{E}$ & 1 & 30 & $\mathrm{NF}$ & 3600 & 144.4203 \\
\hline $\begin{array}{l}1 \\
42\end{array}$ & & 2512 & 257 & 73.7990 & 74.2 & $6.52 \mathrm{E}$ & 267 & 73.7990 & 78.6 & & 1 & 30 & NF & 3600 & 120.4264 \\
\hline 43 & & 2437 & 260 & 77.9 & & & 249 & 79.3 & & & 1 & 30 & NF & 3600 & 124.2335 \\
\hline 44 & & & 25 & & & & 247 & & & & 1 & & & & \\
\hline 45 & & 2503 & 253 & 75.2203 & 76.7672 & $1.46 \mathrm{E}+00$ & 258 & 78.0000 & $\begin{array}{l}81.0022 \\
80.2107\end{array}$ & $1.93 \mathrm{E}$ & 1 & 300 & $\mathrm{NF}$ & 3600 & $\begin{array}{l}126.097 \\
126.998\end{array}$ \\
\hline
\end{tabular}

these cost functions. Note that GALS has superior or equal performance than that of the standard GA on all instances using cost functions $f_{2 a}, f_{2 b}$ and $f_{2 c}$.

In order to show the convergence speed of GALS compared to the standard GA, the convergence graphs for the large-sized problems are presented in Fig. 7. Since LindoGlobal was unable to find any feasible solution in the first 300 seconds, the result by LindoGlobal is not included in Fig. 7. As can be seen in Fig. 7, GALS is able to converge faster and find better quality solutions than those of the standard GA (Fig. 7).

\section{Conclusion}

This paper proposes a hybrid genetic algorithm with local search (GALS) for solving a single-source single-sink MCFP. Since many real-world MCFPs cannot be adequately formulated using linear and convex cost functions, in this paper a general nonlinear non-convex single-source single-sink MCFP is considered. The proposed GALS method is compared with the standard GA, and a mathematical solver LindoGlobal. The proposed algorithm has been evaluated on a set of 45 small, medium and large-sized MCFP instances. Our experi- 
Table 3: Results of GALS, GA, and LindoGlobal using cost function $f_{2 b}$.

\begin{tabular}{|c|c|c|c|c|c|c|c|c|c|c|c|c|c|c|c|}
\hline \multicolumn{16}{|c|}{ Small and medium-sized instances } \\
\hline No. & Nodes & Arcs & \multicolumn{4}{|c|}{ GALS } & \multicolumn{4}{|c|}{$\frac{\mathrm{GA}}{\text { mean }}$} & & \multicolumn{4}{|c|}{ LINDOGlobal } \\
\hline No. & \multirow{6}{*}{5} & Arcs & $\mathrm{t}$ & $\mathrm{b}_{\mathrm{D}}$ & mean & std & $\mathrm{t}$ & D & mean & std & & $\mathrm{t1}$ & & & $\mathrm{Obj} 2$ \\
\hline 1 & & 7 & 16 & 26.0000 & 26.0000 & $1.29 \mathrm{E}-15$ & 18 & 26.0000 & 26.0000 & $6.19 \mathrm{E}-15$ & 0 & - & & 1 & 26.0000 \\
\hline 2 & & 7 & 16 & 26.0000 & 26.0000 & 3.21E-15 & 17 & 26.0000 & 26.0000 & $3.39 \mathrm{E}-15$ & 0 & - & - & 2 & 26.0000 \\
\hline 3 & & 8 & 16 & 26.0000 & 26.0000 & $8.30 \mathrm{E}-15$ & 16 & 26.0000 & 26.0000 & $4.70 \mathrm{E}-15$ & 0 & - & - & 4 & 26.0000 \\
\hline 4 & & 10 & 18 & 26.0000 & 26.0000 & $6.29 \mathrm{E}-15$ & 17 & 26.0000 & 26.0000 & $5.14 \mathrm{E}-15$ & 0 & - & - & 6 & 26.0000 \\
\hline 5 & & 8 & 17 & 26.0000 & 26.0000 & $6.30 \mathrm{E}-15$ & 18 & 26.0000 & 26.0000 & $8.22 \mathrm{E}-15$ & 0 & - & - & 3 & 26.0000 \\
\hline & & 32 & 21 & 52.0000 & 52.0000 & $1.36 \mathrm{E}-14$ & 22 & 52.0000 & 52.0000 & $2.22 \mathrm{E}-14$ & 0 & - & - & 60 & 52.0000 \\
\hline 7 & & 25 & 20 & 52.0000 & 52.0000 & $1.40 \mathrm{E}-14$ & 20 & 52.0000 & 52.0000 & $1.36 \mathrm{E}-14$ & 0 & - & - & 25 & 52.0000 \\
\hline 8 & 10 & 34 & 24 & 52.0000 & 52.0000 & $2.33 \mathrm{E}-14$ & 20 & 52.0000 & 52.0000 & $1.28 \mathrm{E}-14$ & 0 & - & 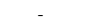 & 40 & 52.0000 \\
\hline 9 & & 20 & 22 & 52.0000 & 52.0000 & $3.50 \mathrm{E}-14$ & 25 & 52.0000 & 52.0000 & 4.40E-14 & 0 & - & - & 23 & 52.0000 \\
\hline 10 & & 32 & 23 & 52.0000 & 52.0000 & $2.36 \mathrm{E}-14$ & 24 & 52.0000 & 52.0000 & $3.26 \mathrm{E}-14$ & 0 & 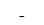 & & 185 & 52.0000 \\
\hline 11 & & 123 & 58 & 52.0000 & 52.0000 & $2.42 \mathrm{E}-14$ & 59 & 52.0000 & 52.0000 & $3.43 \mathrm{E}-14$ & 0 & 300 & 52.0000 & 3600 & 52.0000 \\
\hline 12 & & 142 & 61 & 52.0000 & 52.0000 & $2.46 \mathrm{E}-15$ & 60 & 52.0000 & 52.0000 & 3.42E-15 & 0 & 300 & 52.0000 & 3600 & 52.0000 \\
\hline 13 & 20 & 88 & 61 & 52.0000 & 52.0000 & $1.36 \mathrm{E}-14$ & 57 & 52.0000 & 52.0000 & 1.25E-14 & 0 & 300 & 52.0000 & 3600 & 52.0000 \\
\hline 14 & & 133 & 72 & 109.5208 & 110.5929 & $2.40 \mathrm{E}+00$ & 73 & 110.1137 & 114.4523 & $3.52 \mathrm{E}+00$ & 1 & 300 & 121.4710 & 3600 & 121.4711 \\
\hline 15 & & 117 & 67 & 101.5848 & 102.2805 & 7.15E-01 & 65 & 101.5848 & 105.4304 & $2.53 \mathrm{E}+00$ & 1 & 300 & 115.6350 & 3600 & 115.6345 \\
\hline 16 & & 363 & 86 & 78.0000 & 78.0000 & $1.46 \mathrm{E}-14$ & 81 & 78.0000 & 78.0000 & $2.47 \mathrm{E}-12$ & 0 & 300 & $\mathrm{NF}$ & 3600 & 78.0000 \\
\hline 17 & & 295 & 78 & 78.0000 & 78.0000 & $3.08 \mathrm{E}-12$ & 76 & 78.0000 & 78.0000 & $0.00 \mathrm{E}+00$ & 0 & 300 & 78.0000 & 3600 & 78.0000 \\
\hline 18 & 40 & 320 & 79 & 78.0000 & 78.0000 & $3.02 \mathrm{E}-12$ & 81 & 78.0000 & 78.0000 & $2.67 \mathrm{E}-12$ & 0 & 300 & $\mathrm{NF}$ & 3600 & 78.0000 \\
\hline $\begin{array}{l}18 \\
19\end{array}$ & & $\begin{array}{l}320 \\
343\end{array}$ & 85 & 78.0000 & 78.0000 & $\begin{array}{l}.026 \mathrm{E}-14 \\
1.0\end{array}$ & 87 & 78.0000 & 78.0000 & $1.46 \mathrm{E}-14$ & 0 & 300 & $\mathrm{NF}$ & 3600 & 78.0000 \\
\hline \multirow{2}{*}{\multicolumn{16}{|c|}{$\begin{array}{c}\text { E-12 } 88 \text { - } 88.0000 \\
\text { Large-sized instances }\end{array}$}} \\
\hline & & & & & & & & & & & & & & & \\
\hline 21 & & 844 & 98 & 95.3350 & 95.7707 & $6.37 \mathrm{E}-01$ & 91 & 96.1563 & 98.2845 & $\overline{8+00}$ & 1 & 300 & $\overline{\mathrm{NF}}$ & 3600 & 114.0843 \\
\hline $\begin{array}{l}21 \\
22\end{array}$ & & & 105 & & & $\begin{array}{l}0.34 \\
1.42\end{array}$ & $\begin{array}{l}91 \\
115\end{array}$ & & & & 1 & & $\mathrm{NF}$ & 3600 & $\begin{array}{l}114.0043 \\
94.7421\end{array}$ \\
\hline 23 & 60 & & 94 & 86 & $\begin{array}{l}90.2 \\
88.2\end{array}$ & & 95 & & & & 1 & 500 & $\mathrm{NF}$ & $\begin{array}{l}3000 \\
3600\end{array}$ & $\begin{array}{l}94.1421 \\
110.4345\end{array}$ \\
\hline 24 & & & 106 & & & & 108 & & & & 1 & 300 & $N \mathrm{NI}$ & 3600 & 117.5056 \\
\hline 25 & & & 109 & & & & 102 & & & & 1 & 30 & NF & 3600 & 165.5401 \\
\hline 26 & & & 149 & & & & 147 & & & & 1 & & & 3600 & $\mathrm{NF}$ \\
\hline 27 & & & 138 & & & & 14 & & & & 1 & & & & \\
\hline 28 & 70 & $\begin{array}{l}1103 \\
1231\end{array}$ & 138 & & & & 141 & & & & 1 & & $\mathrm{NF}$ & 3600 & $\mathrm{FF}$ \\
\hline 29 & & & 134 & & & & 131 & & & & 1 & & NF & 3600 & $\mathrm{NF}$ \\
\hline 30 & & 1252 & 151 & & & & 155 & & & & 1 & 30 & NF & 3600 & $\mathrm{NF}$ \\
\hline 31 & & 17 & 165 & & & & 170 & & & & 1 & & & 3600 & \\
\hline 32 & & & 160 & 86.4 & & & 17 & & & & 1 & & & & \\
\hline $\begin{array}{l}32 \\
33\end{array}$ & 80 & 1513 & 165 & 87 & 87 & & 154 & & & & 1 & & & $\begin{array}{l}3000 \\
3600\end{array}$ & $\mathrm{NF}$ \\
\hline 34 & & 18 & $\begin{array}{l}160 \\
169\end{array}$ & 86 & & & 17 & & & & 1 & & $\mathrm{NF}$ & $\begin{array}{l}3000 \\
3600\end{array}$ & F \\
\hline $\begin{array}{l}34 \\
35\end{array}$ & & $\begin{array}{l}1880 \\
1619\end{array}$ & 155 & $\begin{array}{l}80 \\
82\end{array}$ & $\begin{array}{l}8 \\
8\end{array}$ & & 163 & $8:$ & $8 !$ & 5 & 1 & ) & $\mathrm{NF}$ & $\begin{array}{l}3000 \\
3600\end{array}$ & TF \\
\hline 36 & & $18 !$ & 166 & 80.8 & 8 & & 169 & 82 & 9 & 5 & 1 & 30 & $\mathrm{NF}$ & 3600 & \\
\hline 37 & & & 19 & & & & 1 s. & & & & 1 & & & & \\
\hline 38 & 90 & 21 & 202 & 88 & 89.2 & & 1 s. & 88 & & & 1 & ) & $\mathrm{NF}$ & 3600 & $\mathrm{NF}$ \\
\hline 39 & & & 188 & 83 & & & 18 & & & & 1 & & & 3600 & $\mathrm{NF}$ \\
\hline $\begin{array}{l}39 \\
40\end{array}$ & & $\begin{array}{l}1944 \\
2013\end{array}$ & $\begin{array}{l}188 \\
195\end{array}$ & $\begin{array}{l}83.6 \\
82.8\end{array}$ & 8 & $\begin{array}{l}1.38 \\
2.38\end{array}$ & $\begin{array}{l}189 \\
200\end{array}$ & $\begin{array}{l}83.6 \\
83.6\end{array}$ & $\begin{array}{l}90.0884 \\
90.0845\end{array}$ & $\begin{array}{l}5.22 \\
2.90\end{array}$ & $\begin{array}{l}1 \\
1\end{array}$ & 300 & $\mathrm{NF}$ & $\begin{array}{l}\begin{array}{l}50000 \\
3600\end{array}\end{array}$ & $\mathrm{TF}$ \\
\hline 41 & & 250 & 251 & 2.15 & & & 229 & & 95.8 & & & & & 3600 & \\
\hline 42 & & & 27 & & & & 26 & & & & & & & & \\
\hline 43 & 100 & & 278 & & & & 26 & & & & 1 & & & 3600 & \\
\hline 44 & & & 285 & & & & 28 & & & & 1 & 300 & & 3600 & \\
\hline 45 & & 2503 & 286 & 95.3350 & 125 & $1.48 \mathrm{E}+00$ & 265 & 95.3350 & 104. & $.78 \mathrm{E}+00$ & 1 & 300 & NF & 3600 & $\mathrm{NF}$ \\
\hline
\end{tabular}

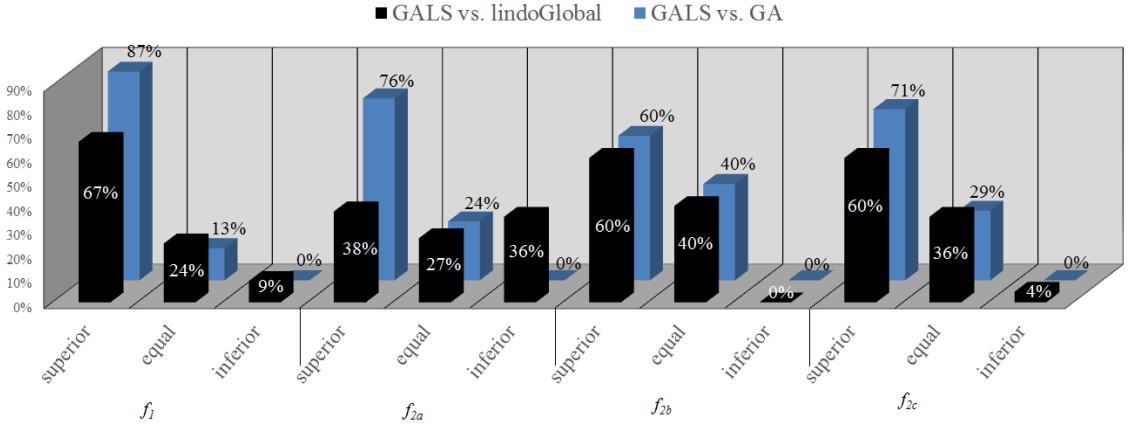

Fig. 6: The number of "wins-draws-loses" of GALS as compared with that of standard GA and LindoGlobal using cost functions $f_{1}, f_{2 a}, f_{2 b}$ and $f_{2 c}$.

mental results show that GALS method significantly outperforms the standard GA and LindoGlobal in terms of solution quality and convergence speed. It is clearly evident that GALS method can handle the large-sized MCFP instances more effectively and efficiently. In contrast, LindoGlobal could not find any feasible solutions for large-sized problems using cost function $f_{1}, f_{2 b}$ and $f_{2 c}$. In 
Table 4: Results of GALS, GA, and LindoGlobal using cost function $f_{2 c}$.

\begin{tabular}{|c|c|c|c|c|c|c|c|c|c|c|c|c|c|c|c|}
\hline \multicolumn{16}{|c|}{$\begin{array}{l}\text { Small and medium-sized instances } \\
\end{array}$} \\
\hline No. & Nodes & Arcs & & & GALS & & & & & & $h$ & & 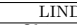 & Globa & \\
\hline & \multirow{5}{*}{5} & & $\mathrm{t}$ & $\frac{\mathrm{b}}{\mathrm{b}}$ & mean & std & $\mathrm{t}$ & $\mathrm{b}$ & mean & std & & t1 & Obj1 & t2 & $\mathrm{Obj} 2$ \\
\hline $\begin{array}{l}1 \\
2\end{array}$ & & $\begin{array}{l}7 \\
7\end{array}$ & $\begin{array}{l}19 \\
17\end{array}$ & $\begin{array}{l}17.7868 \\
20.7513\end{array}$ & $\begin{array}{l}17.7868 \\
20.7513\end{array}$ & $\begin{array}{l}3.54 \mathrm{E}-14 \\
3.91 \mathrm{E}-14\end{array}$ & $\begin{array}{l}20 \\
18\end{array}$ & $\begin{array}{l}26.0000 \\
26.0000\end{array}$ & $\begin{array}{l}17.7868 \\
20.7513\end{array}$ & $\begin{array}{l}0.00 \mathrm{E}+00 \\
0.00 \mathrm{E}+00\end{array}$ & $\begin{array}{l}0 \\
0\end{array}$ & - & - & $\begin{array}{l}1 \\
1\end{array}$ & $\begin{array}{l}17.7868 \\
20.7513\end{array}$ \\
\hline 3 & & 8 & 17 & 17.7868 & 17.7868 & $\begin{array}{l}3.91 \mathrm{E}-14 \\
2.48-10\end{array}$ & $\begin{array}{l}18 \\
18\end{array}$ & 26.0000 & 17.7868 & $0.00 \mathrm{E}+00$ & 0 & $=$ & $=$ & ${ }_{2}^{1}$ & 17.7868 \\
\hline 4 & & 10 & 19 & 20.7513 & 20.7513 & $1.56 \mathrm{E}-09$ & 15 & 26.0000 & 20.7513 & $0.00 \mathrm{E}+00$ & 0 & - & & 29 & 20.7513 \\
\hline 5 & & 8 & 18 & 17.7868 & 17.7868 & $3.85 \mathrm{E}-11$ & 16 & 26.0000 & 17.7868 & $0.00 \mathrm{E}+00$ & 0 & & & 2 & 17.7868 \\
\hline 6 & \multirow{5}{*}{10} & 32 & 27 & 28.9157 & 32.7254 & $2.91 \mathrm{E}+00$ & 27 & 35.0294 & 35.6087 & $1.19 \mathrm{E}+00$ & 1 & 300 & 32.2010 & 3600 & 32.2010 \\
\hline 7 & & 25 & 22 & 31.9726 & 31.9726 & $1.09 \mathrm{E}-14$ & 27 & 31.9726 & 31.9726 & $1.09 \mathrm{E}-14$ & 0 & 300 & 31.9729 & 3600 & 31.9729 \\
\hline 8 & & 34 & 29 & 32.2010 & 32.2010 & $0.00 \mathrm{E}+00$ & 29 & 35.0294 & 35.1480 & $2.43 \mathrm{E}-01$ & 1 & 300 & 32.2010 & 3600 & 32.2010 \\
\hline 9 & & 20 & 35 & 32.2010 & 32.2010 & $0.00 \mathrm{E}+00$ & 38 & 43.6508 & 43.6508 & $0.00 \mathrm{E}+00$ & 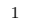 & 300 & 32.2010 & 3600 & 32.2010 \\
\hline 10 & & 32 & 31 & 32.2010 & 32.2010 & 1.46E-14 & 34 & 32.2010 & 32.2010 & 1.46E-14 & 0 & 300 & 32.2010 & 3600 & 32.2010 \\
\hline 11 & \multirow{5}{*}{20} & 123 & 68 & 32. & 32 & 5 & 65 & 32.2010 & 33 & & & 30 & 32.2010 & 3600 & 32.2010 \\
\hline 12 & & 142 & 73 & 35.0 & 35 & & 74 & & & & & 300 & 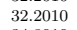 & & 32.2010 \\
\hline 13 & & 88 & 70 & 34.2081 & 34.9881 & $1.06 \mathrm{I}$ & 71 & & 39.4494 & & 1 & 00 & 34.8010 & 00 & 34.8010 \\
\hline 14 & & 133 & 74 & 65.5442 & 65.5442 & $2.92 \mathrm{E}-14$ & 75 & 66.9584 & 44 & $1.32 \mathrm{E}+00$ & 1 & 300 & 108.5580 & 3600 & 81.8294 \\
\hline 15 & & 117 & 67 & 73.8934 & 73.8934 & 1.46E-14 & 77 & 73.8934 & 75.5562 & $1.40 \mathrm{E}+00$ & 1 & 300 & 93.6000 & 3600 & 72.6152 \\
\hline 16 & \multirow{5}{*}{40} & 363 & 77 & 38.4020 & 38.4020 & $1.46 \mathrm{E}-14$ & 70 & 38.4020 & 38.9767 & $2.14 \mathrm{E}+00$ & 0 & 300 & NF & 3600 & 38.4020 \\
\hline 17 & & 295 & 65 & 38.4020 & 38.4020 & $1.46 \mathrm{E}-14$ & 67 & 38.4020 & 38.4020 & $1.46 \mathrm{E}-14$ & 0 & 300 & $\mathrm{NF}$ & 3600 & 38.4020 \\
\hline 18 & & 320 & 71 & 38.4020 & 38.4020 & $1.46 \mathrm{E}-14$ & 72 & 38.4020 & 38.5024 & $2-01$ & 0 & 300 & $\mathrm{NF}$ & 3600 & 38.4020 \\
\hline 19 & & 343 & 78 & 38.4020 & 38.7031 & 9.821 & 74 & 38.4020 & 38.6027 & 6.18 & 0 & 300 & $\mathrm{NF}$ & 3600 & 38.4020 \\
\hline 20 & & 294 & 72 & 38.4020 & 38.4020 & $1.46 \mathrm{E}-14$ & 74 & 38.4020 & 39.0042 & $1.47 \mathrm{E}+00$ & & 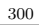 & $\mathrm{NF}$ & 3600 & 38.4020 \\
\hline & \multicolumn{15}{|c|}{ ed instances } \\
\hline 21 & \multirow{5}{*}{60} & 844 & 100 & & & $5.75 \mathrm{E}-01$ & 109 & & & & & & $\overline{\mathrm{NH}}$ & & 553 \\
\hline 22 & & 90 & & & & & 112 & & & & & & & & \\
\hline 23 & & 79 & & 61. & & & 10 & & & & & & & & \\
\hline 24 & & 91 & & & & & 12 & & & & & & $\mathrm{~N}$ & & \\
\hline 25 & & 87 & 4 & 62. & & & 107 & & & & & 30 & $\mathrm{NH}$ & 00 & 219.4061 \\
\hline 26 & \multirow{5}{*}{70} & 1176 & 135 & & 59. & & 110 & 61. & 62. & & 1 & 30 & $\mathrm{NF}$ & 3600 & 77.7716 \\
\hline 27 & & 1163 & 120 & & & & 116 & & & & 0 & & NF & & 0.4071 \\
\hline 28 & & 12 & 141 & & & & 148 & & & & 1 & & $\mathrm{NF}$ & & 75.1716 \\
\hline 29 & & 99 & 124 & & & & 121 & & & & 1 & 30 & $\mathrm{NF}$ & 3600 & 90.4071 \\
\hline 30 & & 1252 & 148 & & 60.2 & & 149 & & & & 1 & 300 & $\mathrm{NF}$ & 3600 & 91.5929 \\
\hline 31 & \multirow{5}{*}{80} & & 15 & & & & 14 & & & & 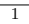 & & $\mathrm{NF}$ & 0 & $\mathrm{NF}$ \\
\hline 32 & & & 15 & 58.2 & & & 16 & & & & 1 & & $\mathrm{~N}$ & & \\
\hline $\begin{array}{l}32 \\
33\end{array}$ & & 1513 & 147 & 58.2 & 5 & 2. & 16 & 63. & 66 & 2. & 1 & & $\mathrm{~N}$ & 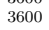 & $\mathrm{F}$ \\
\hline 34 & & 1880 & 166 & 60.8 & 6 & & 160 & 63. & 66 & 2. & 1 & 30 & $\mathrm{NF}$ & 0 & $F$ \\
\hline 35 & & 1619 & 155 & & & & 165 & & & & 1 & 30 & NF & 0 & 186.3787 \\
\hline 36 & \multirow{5}{*}{90} & & 165 & & & & 16 & & & & & & & & \\
\hline 37 & & & & & & & & & & & & & & & \\
\hline 38 & & & 26 & & & & & & & & & & $\mathrm{~N}$ & & \\
\hline 39 & & & & & & & & & & & 1 & & & & \\
\hline 40 & & & 2 & & & & 21 & & & & 1 & & $\mathrm{NF}$ & & 239.2975 \\
\hline 41 & \multirow{5}{*}{100} & & & & & & 24 & & & & 1 & 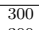 & & & \\
\hline 42 & & & & 65.8 & & & 25 & & 69. & 3.0 & 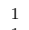 & 20 & $\mathrm{NF}$ & 36 & $\mathrm{NI}$ \\
\hline 43 & & 2437 & 261 & 58.26 & 59.3 & 1.6 & 263 & 58.2 & 64. & 4.03 & 1 & 300 & $\mathrm{NF}$ & 3600 & $\mathrm{NF}$ \\
\hline 44 & & 2370 & 264 & 60.8010 & 61.8527 & $1.68 \mathrm{E}+00$ & 271 & 61.0294 & 65.4772 & $3.29 \mathrm{E}+00$ & 1 & 300 & $\mathrm{NF}$ & 3600 & NF \\
\hline 45 & & 2503 & 274 & 61.6223 & 62.5712 & $1.16 \mathrm{E}+00$ & 278 & 70.2437 & 71.9140 & $1.65 \mathrm{E}+00$ & 1 & 300 & $\mathrm{NF}$ & 3600 & $\mathrm{NF}$ \\
\hline
\end{tabular}

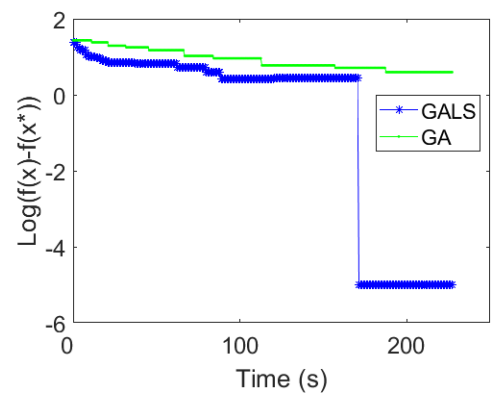

(a) $f_{2 a}$ on instance No.45

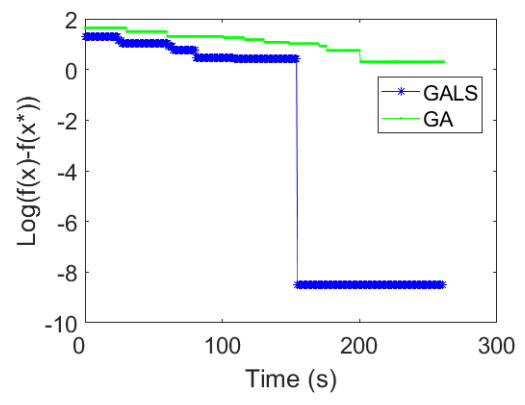

(b) $f_{2 c}$ on instance No.40

Fig. 7: Convergence plot for the proposed GALS method and standard GA.

addition, GALS can handle very well the selected nonlinear non-convex cost functions, whereas existing mathematical solvers are too sensitive to the shape of the function. This robustness property is an important strength of the GA- 
based methods in solving MCFPs. Our future work will examine performance of GALS method on practical telecommunication network problems.

\section{Compliance with Ethical Standards}

Conflict of Interest: The authors declare that they have no conflict of interest.

Ethical approval: This article does not contain any studies with human participants or animals performed by any of the authors.

\section{References}

1. Ahuja, R.K., Magnanti, T.L., and Orlin, J.B., Network flows: theory, algorithms, and applications,Prentice hall , pp.4-6, (1993).

2. Vegh, Laszlo A. A strongly polynomial algorithm for a class of minimum-cost flow problems with separable convex objectives." SIAM Journal on Computing 45.5 (2016): 17291761.

3. Yan, S., Y. L. Shih, and C. L. Wang. An ant colony system-based hybrid algorithm for square root concave cost transhipment problems. Engineering Optimization 42.11 (2010): 983-1001.

4. Lin, ShinYeu, and ChiHsin Lin. A computationally efficient method for nonlinear multicommodity network flow problems. Networks: An International Journal 29.4 (1997): $225-244$.

5. Newell, G. F. Non-convex traffic assignment on a rectangular grid network. Transportation science 30.1 (1996): 32-42.

6. Garey, Michael R., and David S. Johnson. Computers and intractability: A guide to the theory of npcompleteness (series of books in the mathematical sciences), ed. Computers and Intractability 340 (1979).

7. Guisewite, Geoffrey M., and Panos M. Pardalos. Algorithms for the single-source uncapacitated minimum concave-cost network flow problem. Journal of Global Optimization 1.3 (1991): 245-265.

8. Monteiro, Marta SR, Dalila BMM Fontes, and Fernando ACC Fontes. Concave minimum cost network flow problems solved with a colony of ants. Journal of Heuristics 19.1 (2013): $1-33$.

9. Kim, Hak-Jin, and John N. Hooker. Solving fixed-charge network flow problems with a hybrid optimization and constraint programming approach. Annals of Operations Research 115.1-4 (2002): 95-124.

10. Ortega, Francisco, and Laurence A. Wolsey. A branchandcut algorithm for the singlecommodity, uncapacitated, fixedcharge network flow problem. Networks: An International Journal 41.3 (2003): 143-158.

11. Fontes, Dalila BMM, Eleni Hadjiconstantinou, and Nicos Christofides. A branch-andbound algorithm for concave network flow problems. Journal of Global Optimization 34.1 (2006): 127-155.

12. Guisewite, Geoffrey M., and Panos M. Pardalos. Global search algorithms for minimum concave-cost network flow problems. Journal of Global Optimization 1.4 (1991): 309-330.

13. Horst, Reiner, and Nguyen V. Thoai. An integer concave minimization approach for the minimum concave cost capacitated flow problem on networks. Operations-ResearchSpektrum 20.1 (1998): 47-53.

14. Burkard, Rainer E., Helidon Dollani, and Phan Thien Thach. Linear approximations in a dynamic programming approach for the uncapacitated single-source minimum concave cost network flow problem in acyclic networks. Journal of Global Optimization 19.2 (2001): 121-139. 
15. Fontes, Dalila BMM, Eleni Hadjiconstantinou, and Nicos Christofides. A dynamic programming approach for solving single-source uncapacitated concave minimum cost network flow problems. European Journal of Operational Research 174.2 (2006): 1205-1219.

16. Erickson, Ranel E., Clyde L. Monma, and Arthur F. Veinott Jr. Send-and-split method for minimum-concave-cost network flows. Mathematics of Operations Research 12.4 (1987): 634-664.

17. Kovacs, Peter. Minimum-cost flow algorithms: an experimental evaluation. Optimization Methods and Software 30.1 (2015): 94-127.

18. Monteiro, Marta SR, Dalila BMM Fontes, and Fernando ACC Fontes. An ant colony optimization algorithm to solve the minimum cost network flow problem with concave cost functions. Proceedings of the 13th annual conference on Genetic and evolutionary computation (2011): 139-146.

19. Fontes, Dalila BMM, and Jos Fernando Gonalves. Heuristic solutions for general concave minimum cost network flow problems. Networks: An International Journal 50.1 (2007): $67-76$.

20. Xie, Fanrong, and Renan Jia. Nonlinear fixed charge transportation problem by minimum cost flow-based genetic algorithm. Computers and Industrial Engineering 63.4 (2012): 763-778.

21. Reca, Juan, Juan Martnez, and Rafael Lpez-Luque. A new efficient bounding strategy applied to the heuristic optimization of the water distribution networks design. Congress on Numerical Methods in Engineering CMN. 2017.

22. Michalewicz, Zbigniew, George A. Vignaux, and Matthew Hobbs. A nonstandard genetic algorithm for the nonlinear transportation problem. ORSA Journal on computing 3.4 (1991): 307-316.

23. Klansek, Uros, and Mirko Psunder. Solving the nonlinear transportation problem by global optimization. Transport 25.3 (2010): 314-324.

24. Klansek, Uros. Solving the nonlinear discrete transportation problem by MINLP optimization. Transport 29.1 (2014): 1-11.

25. Goldberg, David E., and John H. Holland. Genetic algorithms and machine learning. Machine learning 3.2 (1988): 95-99.

26. Michalewicz, Zbigniew and Hartley, Stephen. Genetic algorithms + data structures $=$ evolution programs. Mathematical Intelligence 18.3 (1996).

27. Cheng, Runwei, and Mitsuo Gen. An evolution programme for the resource-constrained project scheduling problem. International Journal of Computer Integrated Manufacturing 11.3 (1998): 274-287.

28. Gen, Mitsuo, Runwei Cheng, and Dingwei Wang. Genetic algorithms for solving shortest path problems. Evolutionary Computation, 1997., IEEE International Conference on. IEEE, 1997.

29. Lin, Lin, and Mitsuo Gen. Multiobjective Genetic Algorithm for Bicriteria Network Design Problems. Intelligent and Evolutionary Systems. Springer, Berlin, Heidelberg, 2009. $141-161$.

30. Gen, Mitsuo, Runwei Cheng, and Lin Lin. Network models and optimization: Multiobjective genetic algorithm approach. Springer Science and Business Media, 2008.

31. Burer, Samuel, and Adam N. Letchford. Non-convex mixed-integer nonlinear programming: A survey. Surveys in Operations Research and Management Science 17.2 (2012): 97-106.

32. Tawarmalani, Mohit, Nikolaos V. Sahinidis, and Nikolaos Sahinidis. Convexification and global optimization in continuous and mixed-integer nonlinear programming: theory, algorithms, software, and applications. Springer Science \& Business Media (65), (2002).

33. Sherali, Hanif D., and Warren P. Adams. A reformulation-linearization technique for solving discrete and continuous nonconvex problems. Vol. 31. Springer Science and Business Media, 2013.

34. Tawarmalani, Mohit, Nikolaos V. Sahinidis, and Nikolaos Sahinidis. Convexification and global optimization in continuous and mixed-integer nonlinear programming: theory, algorithms, software, and applications. Vol. 65. Springer Science and Business Media, 2002.

35. Lin, Youdong, and Linus Schrage. The global solver in the LINDO API. Optimization Methods and Software 24.4-5 (2009): 657-668.

36. Sinha, Ankur, Pekka Malo, and Kalyanmoy Deb. Evolutionary algorithm for bilevel optimization using approximations of the lower level optimal solution mapping. European Journal of Operational Research 257.2 (2017): 395-411. 